\title{
OPENTD: PLATAFORMA WEB PARA TELEDIAGNÓSTICO DE EXAMES DE IMAGEM
}

\author{
WEB PLATFORM FOR MEDICAL IMAGES TELEDIAGNOSIS
}

\begin{abstract}
Nícolas Vinícius Rodrigues Veras
Engenheiro biomédico e mestrando no Programa de Pós-Graduação em Ciência, Tecnologia e Inovação pela UFRN. Pesquisador do Laboratório de Inovação Tecnológica em Saúde.

Contato: nicolas.vinicius@lais.huol.ufrn.br.

Paulo Victor de Azevedo Guerra
\end{abstract}

Engenheiro biomédico e especialista em Informática em Saúde pela UFRN. Pesquisador do Laboratório de Inovação Tecnológica em Saúde. Contato: paulo.victor@lais.huol.ufrn.br.

Marcel da Câmara Ribeiro Dantas

Mestre em bioinformática e engenheiro de computação e automação pela UFRN. Pesquisador do Laboratório de Inovação Tecnológica em Saúde. Contato: ribeirodantasdm@gmail.com.

\section{Natanael de Freitas Neto}

Engenheiro eletricista pela UFG e mestrando no Programa de Pós-Graduação em Ciência, Tecnologia e Inovação pela UFRN. Pesquisador do Laboratório de Inovação Tecnológica em Saúde.

Contato: natanael.neto@lais.huol.ufrn.br.

Isabele Magaldi Almeida de Freitas

Engenheira biomédica e mestranda no Programa de Pós-Graduação em Engenharia de Produção pela UFRN. Pesquisadora do Laboratório de Inovação Tecnológica em Saúde.

Contato: isabele.magaldi@lais.huol.ufrn.br.

Custódio Leopoldino de Brito Guerra Neto

Doutor em Engenharia de Materiais pela UFRN. Pesquisador do Laboratório de Inovação Tecnológica em Saúde. Contato: custodioimplante@gmail.com.

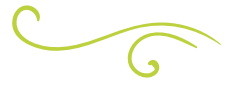

\section{RESUMO}

Na perspectiva de garantir a todos os brasileiros o cumprimento dos princípios básicos do Sistema Único de Saúde (SUS), é importante perceber que a integralidade e a universalidade encontram-se comprometidas devido à dificuldade em atender a demandas e particularidades de um país de dimensões territoriais continentais. A desigualdade no acesso aos serviços de saúde provocada pela carência e má distribuição geográfica de profissionais de saúde em áreas remotas, principalmente no campo da radiologia, dificultam a execução de uma política de saúde tão grande e ambiciosa quanto a do SUS. Nesse contexto, este estudo apresenta uma solução para essa problemática, oferecendo a oportunidade de todo o processo de laudagem ser feito de maneira descentralizada e a distância via web, por meio de uma plataforma de telediagnóstico. Com relação à metodologia, para o desenvolvimento da referida plataforma as seguintes tecnologias foram utilizadas: o sistema de gerenciamento de banco de dados PostgresSOL, por sua 
grande estabilidade e confiabilidade, e o Laravel, que é um framework de desenvolvimento web. Como resultados alcançados, observou-se que, com a implementação do OpenTeleDiagnóstico (OpenTD), é possível transpor barreiras importantes na área da radiologia, impactando de modo positivo o processo de trabalho hospitalar. De fato, os usuários do OpenTD no HUOL mostraram-se satisfeitos com a plataforma. Outro fator significativo constatado no que tange à plataforma é o impacto ambiental e financeiro proporcionado pela redução da utilização de papéis no serviço de saúde, já que os laudos são digitalizados e armazenados de maneira segura.

PALAVRAS-CHAVE: Imagens Médicas. Laudo. Hospital Universitário. Telessaúde. Telediagnóstico.

\section{ABSTRACT}

In the perspective of ensure to all Brazilians the basic principles of the Unified Health System (SUS), integrality and universality are compromised due to the difficulty in meeting the demands and particularities of a country with continental territorial dimensions. The inequality of access to health services caused by the lack of geographical distribution of health professionals in remote areas, especially in the field of radiology, makes it difficult to perform such a large and ambitious health policy. In this context, the study presents a solution to this problem, offering the opportunity of the whole process of medical reporting to be done in a decentralized way and the distance via the web through a telediagnostic platform. Regarding the methodology, the following technologies were used for the development of the mentioned platform: the database management system PostgresSQL, due to its great stability and reliability, and Laravel, which is a web development framework. As results achieved, it was observed with the implementation of the OpenTeleDiagnóstico (OpenTD) that it is possible to overcome important barriers in the area of radiology, positively impacting the hospital work process. OpenTD users at HUOL were satisfied with the platform. Another significant factor observed in the platform is the environmental and financial impact provided by the reduction of paper use in the health service since the reports are digitized and stored safely.

KEYWORDS: Medical Images. Report. University Hospital. Telehealth. Telediagnosis.

\section{INTRODUÇÃO}

Considerado uma das maiores conquistas sociais já vistas na história do Brasil, o Sistema Único de Saúde (SUS), celebrado pela Constituição de 1988, garante a saúde como "direito de todos e dever do estado". Como princípios norteadores dessa política de saúde, estão a universalidade e a descentralização do acesso ao sistema (BRASIL, 2000; FREITAS, 2016). Garantir esse direito a todos os cidadãos é um desafio, tendo em vista as dimensões continentais do Brasil e as diversas faces das desigualdades encontradas no país, sejam elas de cunho social, econômico, político ou geográfico. A complexidade desses obstáculos tem levado a problemas no acesso aos serviços e ao comprometimento da universalidade e integralidade, privando grande parcela da população de usufruir do direito universal à saúde (GUERRA, 2015).

Nesse sentido, há uma disparidade no acesso aos serviços de saúde, provocada pela carência e má distribuição geográfica de médicos radiologistas em áreas remotas. Essa escassez fica clara quando observamos a distribuição desses especialistas por região do Brasil (DANTAS, 2016; SCHEFFER et al., 2018). 


\begin{tabular}{|c|c|}
\hline Norte & $3,4 \%$ \\
\hline Nordeste & $17,8 \%$ \\
\hline Sudeste & $52,9 \%$ \\
\hline Sul & $16,9 \%$ \\
\hline Centro-Oeste & $9,1 \%$ \\
\hline
\end{tabular}

Tabela 1 - Distribuição de radiologistas por região

Fonte: Shreller et al. (2018).

No entanto, os avanços das Tecnologias de Informação e Comunicação (TICs), principalmente na área da saúde, permitiram, por meio da internet, transmitir a informação com mais eficiência entre locais distantes, rompendo as barreiras do tempo e das fronteiras territoriais (MELO; SILVA, 2006). Nesse marco, foi criado pelo Ministério da Saúde, no ano de 2005, o Projeto Telemedicina. Segundo o Conselho Federal de Medicina (CFM), a Telemedicina é "o exercício da Medicina através da utilização de metodologias interativas de comunicação audiovisual e de dados, com o objetivo de assistência, educação e pesquisa em Saúde" (CONSELHO FEDERAL DE MEDICINA, 2002).

Ressalta-se que o Sistema Único de Saúde (SUS) surge quando a Constituição de 1988 estabelece a saúde como direito de todos e dever do Estado. A partir desse momento, ficou definido que o sistema restrito e centralizado daria lugar a um novo modelo que seria conduzido de maneira irrestrita e descentralizada (BRASIL, 2000; FREITAS, 2016). Assim, era necessária uma abordagem diferente da que vinha sendo praticada quando o assunto é saúde.

Conforme o SUS foi evoluindo, as Tecnologias de Informação e Comunicação (TICs) também foram ganhando muito destaque, já que, por meio da internet, a informação é transmitida com mais eficiência entre locais distantes, rompendo as barreiras do tempo e das fronteiras territoriais (MELO; SILVA, 2006).

E foi neste cenário que, no Brasil, o Programa Telessaúde surgiu. Logo, a utilização de serviços de telediagnóstico na medicina é relativamente recente. $O$ primeiro relato da utilização de tecnologias de comunicação para realização de testes diagnósticos foi em 1950, por meio do uso do fax para transmissão de raio $X$ para serviços especializados em radiologia nos Estados Unidos. Entretanto, foi a partir dos anos 1990, com o desenvolvimento da área de telecomunicações, que essa tecnologia ganhou espaço como alternativa para suprir a dificuldade de acesso a exames em áreas remotas. Otelediagnóstico está definido na Portaria do MS n 2.546 como "serviço autônomo que utiliza as tecnologias da informação e comunicação para realizar serviços de apoio ao diagnóstico através de distâncias geográfica e temporal" (BRASIL, 2011).

No SUS, são realizados diariamente milhares de exames de imagem e todos eles precisam de um laudo. Essa necessidade gera um problema por depender exclusivamente da presença de um médico radiologista para redigir e assinar um laudo que será anexado ao exame. Por vezes, esses exames demoram a ser laudados justamente pela falta de um médico no Estabelecimento Assistencial de Saúde (EAS) naquele momento. Com frequência, pacientes aguardam laudos de exames para receber a alta hospitalar e ocupam dezenas de leitos em detrimento de outros pacientes que esperam ansiosamente na longa fila do Sistema Único de Saúde. Isso impacta diretamente na qualidade do atendimento prestado aos usuários, fazendo com que caia o nível de satisfação deles (ADAS; MOIMAZ; SALIBA, 2010).

Além disso, os médicos geralmente dividem seus dias entre diversos hospitais e clínicas, fazendo com que a rotatividade dentro de um hospital seja muito alta. Muitas vezes, isso impede que um mesmo profissional possa acompanhar mais de perto a evolução de um paciente específico (MEIRA, 2004).

Considerando esses problemas, O objetivo deste artigo é apresentar uma plataforma web para laudagem remota de exames de imagem, fazendo, assim, com que o médico possa emitir um laudo de qualquer parte do mundo, desde que tenha acesso à internet. Essa ferramenta poderá 
agilizar o processo, diminuir a fila por laudos e aumentar a qualidade no atendimento.

\section{MATERIAIS E MÉTODOS}

A proposta desta plataforma é possibilitar aos médicos uma melhoria significativa em seu processo de trabalho, uma vez que eles não precisem estar fisicamente no local em que os exames foram realizados. O componente front-end da plataforma foi desenvolvido utilizando as linguagens HTML, CSS e JavaScript. A linguagem utilizada no back-end foi PHP, utilizando também o framework Laravel. O Sistema de Gerenciamento de Banco de Dados escolhido foi o PostegreSQL. Utilizou-se - Git como ferramenta de versionamento de código e o GitLab como interface web para demais interações com o repositório. Todas as funcionalidades foram testadas em servidor local de uma máquina com sistema operacional Ubuntu 16.04, processador Intel Core i3-3227U CPU @ 1.90G Hz, 64 bits e 4,0GB RAM.

Para conseguir uma melhoria contínua nos resultados com o desenvolvimento da plataforma, periodicamente os desenvolvedores do OpenTD se reúnem com uma equipe do HUOL que é composta por físicos médicos, médicos radiologistas e técnicos de radiologia para que eles possam fornecer feedback sobre o uso do mínimo produto viável que está em testes. A partir do retorno e dos dados obtidos com as experiências dos usuários, novas melhorias e modificações são realizadas constantemente.

Cabe salientar que os exames possuirão marcadores quanto ao tempo que estão aguardando para serem laudados. Com isso, será possível gerenciar, através da plataforma, a qualidade do serviço prestado, bem como identificar os gargalos e mensurar novas demandas em decorrência disso. Ainda existirão etiquetas coloridas que indicarão o nível de urgência com que é necessário emitir o laudo de determinado exame. Três níveis de urgência estarão disponíveis no OpenTD: emergência, urgência e eletivo. Dessa forma, os médicos poderão utilizar este parâmetro na hora de priorizar as respostas dos telediagnósticos solicitados.

No módulo de downloads, os usuários da plataforma têm acesso a todos os exames realizados, de modo que poderão fazer o download e analisar cada imagem antes de realizar o laudo. $\bigcirc$ módulo de cadastro é onde o responsável por admitir o paciente no estabelecimento de saúde irá inserir suas informações para que aquele paciente seja um ator na plataforma, além de cadastrar o exame que será realizado posteriormente. O módulo de laudos é onde, de fato, o médico irá emitir os laudos dos exames dos quais realizou o download anteriormente.

A figura 1 representa de maneira clara o fluxo do OpenTD. Quando os exames são realizados, automaticamente essas imagens ficam disponíveis na plataforma que as disponibiliza para radiologistas de todo o Brasil. Quando esses profissionais emitem o laudo, este fica salvo na plataforma junto ao exame.

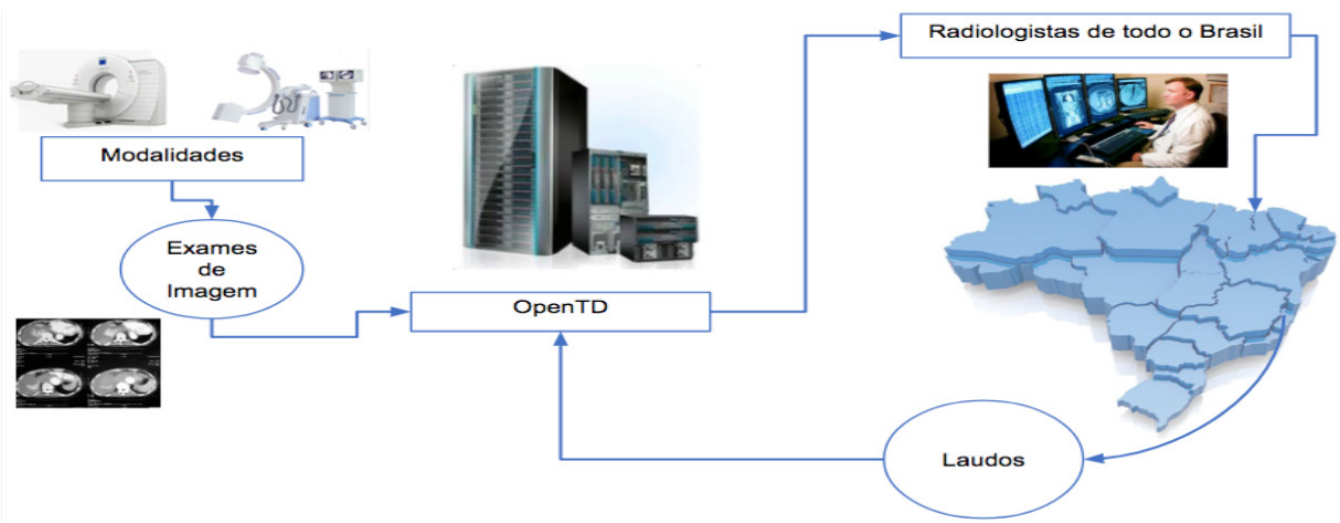

Figura 1 - Fluxo do OpenTD. 


\section{RESULTADOS E DISCUSSÃO}

O desenvolvimento da plataforma encontra-se em fase final e um 'Mínimo Produto Viável (MVP) está sendo utilizado no Hospital Universitário Onofre Lopes (HUOL) para validação. A utilização do OpenTD poderá diminuir o tempo de espera por um laudo de exame de imagem, já que um processo hoje feito à mão passará a ser digitalizado.

Dessa forma, radiologistas de todo o país estarão realizando os laudos desses exames, eliminando fronteiras e possibilitando que habitantes de todas as regiões brasileiras tenham acesso a um médico especialista. Na figura 2, podemos ver a tela inicial, onde o usuário pode solicitar o seu cadastro ou entrar com seu login e senha.

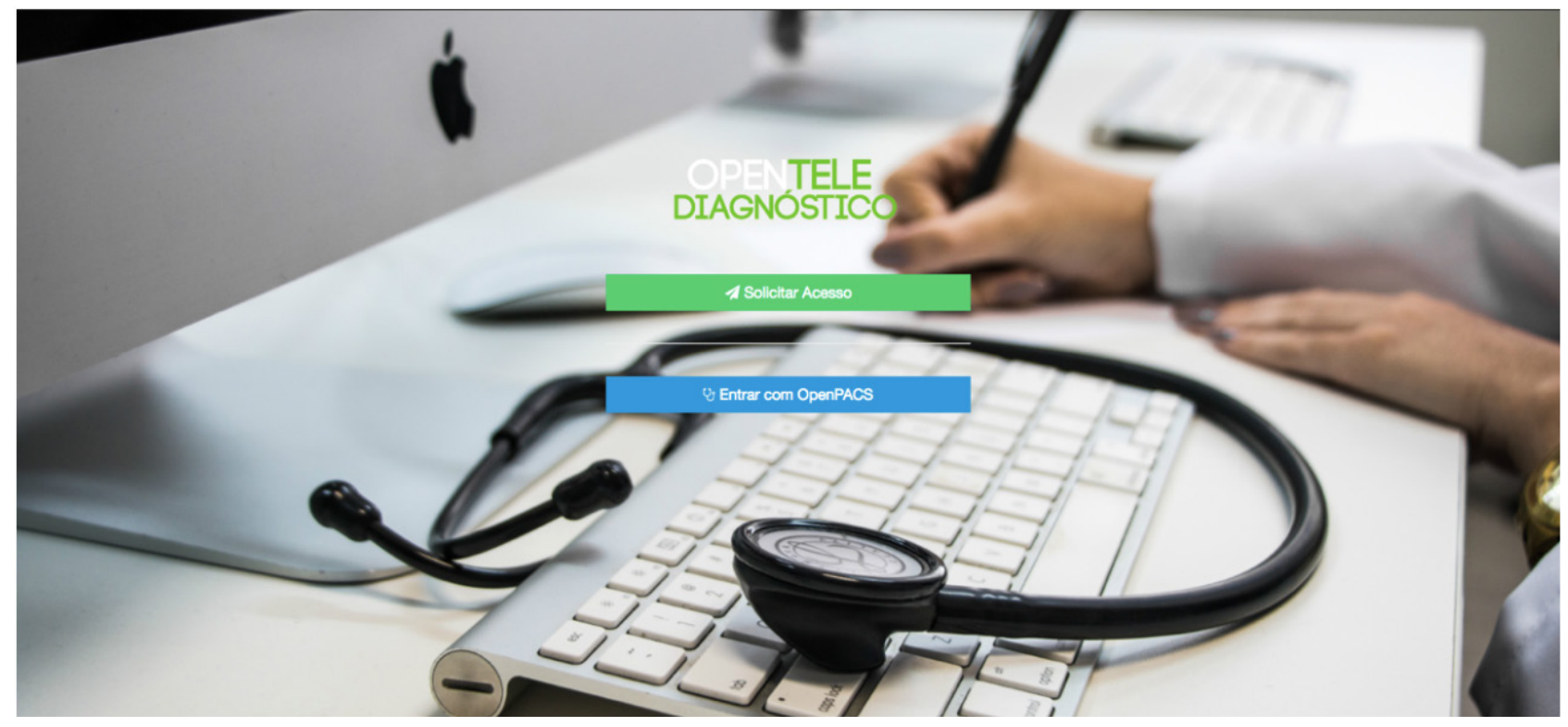

Figura 2 - Página inicial do OpenTD.

\section{DISCUSSÃO}

Fonte: Autoria própria (2018)

Na figura 3 abaixo, está a tela onde é possível cadastrar um novo exame para um paciente que deu entrada no EAS. O fluxo, de fato, se inicia com essa tela, já que o atendente irá digitar o CPF do paciente e, em seguida a plataforma irá informar se o cadastro já foi realizado anteriormente. Caso o cadastro não exista, o atendente irá preencher todo o formulário e finalizar clicando no botão salvar.

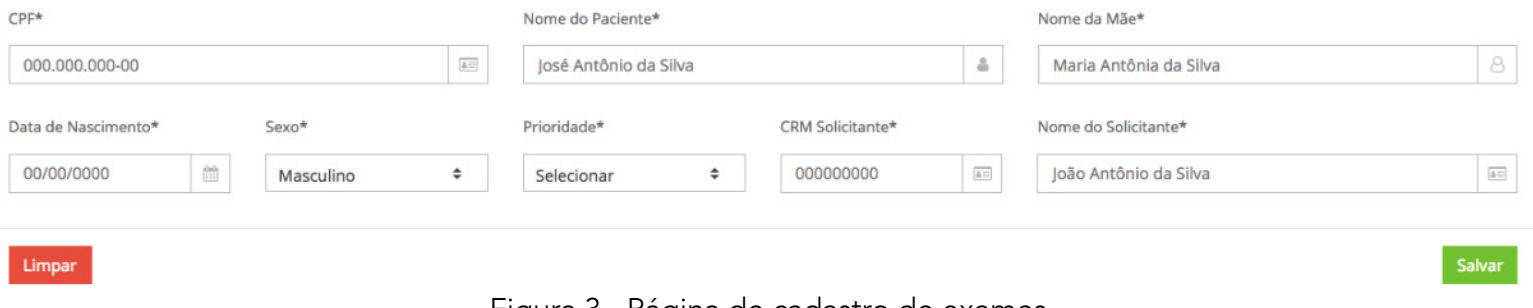

Figura 3 - Página de cadastro de exames. 
Por meio da página de laudos, os médicos podem ver todos os exames que estão na fila e que precisam ser laudados, exibindo informações importantes como a data em que o exame foi realizado e os dados do médico solicitante. Na figura 4, é possível visualizar a tela de laudos, com várias opções de filtros de pesquisa.

\section{OPENTELE}
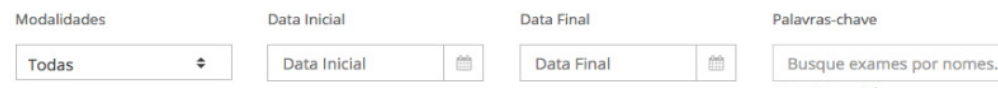

Nome: Qualquer nome Cox Sexo: F/M/O Data Inicial: 17/09/2018 Data Final: 18/09/2018 Modalidade: Todos

Figura 4 - Página contendo a lista de exames que deverão ser laudados.

Fonte: Autoria própria (2018)

Uma importante funcionalidade a que os gestores da plataforma têm acesso é a lista de pacientes, nas quais esses gestores podem consultar quaisquer dados desses pacientes e fazer alguma alteração, caso seja necessária. A plataforma foi desenvolvida visando uma boa experiência do usuário, sendo focada em um uso específico e objetivando usabilidade simples e intuitiva. Por esse motivo, foram desenvolvidos módulos diferentes de pacientes, downloads e laudos. A figura 5 traz a página com a lista de pacientes, também com várias opções de filtros de pesquisa.

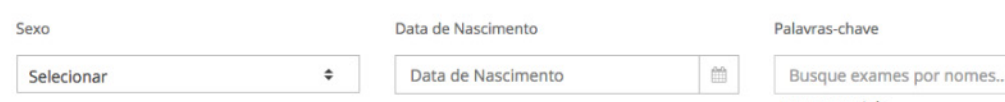

Figura 5 - Página contendo a lista de pacientes.

Outro fator importante de melhoria do OpenTD em relação ao modelo atual é a segurança da assinatura, já que esse processo também ocorre de maneira digital, por meio de criptografia, garantindo que o profissional que está assinando determinado laudo é o indicado. Além disso, há um registro de todas as ações dos usuários na plataforma, sendo possível descobrir qual usuário executa cada ação. 


\section{CONSIDERAÇÕES FINAIS}

Levando-se em conta o objetivo deste estudo, que consiste em apresentar uma plataforma web para laudagem remota de exames de imagem, fazendo, assim, com que o médico possa emitir um laudo de qualquer parte do mundo, desde que tenha acesso à internet, faz-se necessário alinhar os aspectos mais marcantes nesta área de atuação.

Em primeiro lugar, os resultados provenientes da implementação do OpenTeleDiagnóstico como plataforma piloto no HUOL, mostram que é possível transpor barreiras importantes na área da radiologia, impactando de modo positivo o processo de trabalho hospitalar. Assim, tornar essa tecnologia acessível a todos os estabelecimentos de saúde é uma das premissas da Telessaúde como estratégia para atender o princípio da universalidade norteador do Sistema Único de Saúde.

Nesse marco, vale salientar a relevância do OpenTD no contexto do desenvolvimento tecnológico em saúde. A plataforma desenvolvida pelo Laboratório de Inovação Tecnológica em Saúde(LAIS) da Universidade Federal do Rio Grande do Norte (UFRN), localizado no HUOL, permitiu a contribuição de uma equipe transdisciplinar experiente composta por médicos radiologistas, físicos médicos, técnicos de radiologia e desenvolvedores de software, o que resultou em uma plataforma modelada de forma a atender a todas as exigências do serviço, além de sua fácil implementação em outros estabelecimentos. Apesar do módulo de laudagem de exames e a integração ao sistema de assinatura digital ainda estarem em fase final de desenvolvimento e implementação, os usuários do OpenTD no HUOL mostraram-se satisfeitos com a plataforma. Um outro fator significativo no que tange ao OpenTD é o impacto ambiental e financeiro proporcionado pela redução da utilização de papéis no serviço, pois os laudos são digitalizados e armazenados de maneira segura nos servidores da UFRN.
No entanto, é necessário que novas pesquisas, análises e estudos de caso mais aprofundados sejam realizados nos contextos regional e nacional com o intuito de difundir, monitorar e replicar as experiências bem-sucedidas, de modo a confirmar as vantagens da utilização dos serviços de telediagnóstico.

Por fim, conclui-se que o OpenTD é uma contribuição valiosa para a Telessaúde no país, por permitir que os laudos médicos que antes aguardavam horas, dias e até mesmo semanas para serem emitidos, agora sejam realizados rapidamente e com segurança sem a necessidade do profissional radiologista estar presente no ambiente hospitalar. Proporcionou-se também maior qualidade e resolutividade nos serviços de radiologia prestados no $\mathrm{HUOL}$, diminuindo as filas e aumentando a satisfação dos usuários do Sistema Único de Saúde. 


\section{REFERÊNCIAS BIBLIOGRÁFICAS}

ADAS, S.; MOIMAZ, S.; SALIBA, O. Sobre o Serviço Público de Saúde. Physis: Revista de Saúde Coletiva, v. 20, n. 04, p. 1419-1440, 2010.

AL-TAEI, M. K.; PAN, Y.; LU, D. M. An Internet-based telediagnosis system for Chinese medicine. Journal of Telemedicine \& Telecare, v. 6 Suppl 1, p. S63-5, 2000.

BORELLI, J. E.; NEVES, E. D. A.; GONZAGA, A. Telediagnóstico Auxiliado por Computador e Monitoramento Através de Imagens de Reparação Tecidual de Úlceras Tróficas de Perna. Congresso nacional. Jan. 2015, 2000.

BRASIL. Ministério da Saúde. Sistema único de Saúde (SUS): princípios e conquistas. Brasília: Ministério da saúde, 2000.

- Ministério da Saúde. Portaria $n^{\circ} 2.546$, de 27 de outubro de 2011. Redefine e amplia o Programa Telessaúde Brasil, que passa a ser denominado Programa Nacional Telessaúde Brasil Redes (Telessaúde Brasil Redes). 2011.

CONSELHO FEDERAL DE MEDICINA - CFM. Resolução no 1.643, de 7 de agosto de 2002. Define e disciplina a prestação de serviços através da telemedicina., 2002.

DANTAS, M. da C. R. Sistema de Telemonitoramento para Pacientes com Esclerose Lateral Amiotrófica. Universidade Federal do Rio Grande do Norte, 2016.

FREITAS, I. M. A. de. Impactos do programa nacional telessaúde brasil redes na qualidade dos serviços de saúde prestados na atenção básica do estado do rio grande do norte. 2016.

GUERRA, C. L. de B. N. Teleodontologia. In: A Telessaúde no Brasil e a Inovação Tecnológica na Atenção Primária. Natal: EDUFRN, 2015.

MARCOLINO, M. S. et al. A Rede de Teleassistência de Minas Gerais e suas contribuições para atingir os princípios de universalidade, equidade e integralidade do SUS - relato de experiência. Revista Eletrônica de Comunicação, Informação \& Inovação em Saúde, p. v. 7, n. 2, 2013.

MEIRA, A. R. Bioética e vulnerabilidade: o médico e o paciente. Revista da Associação Médica Brasileira, v. 50, n. 3, p. 249-250, 2004.

MELLO, M. R. de et al. Estratégia para Avaliação de um Sistema de Cooperação em Saúde na Web. Jan. 2006, 2015. 
MELO, M. DO C. de; SILVA, E. M. de S. Aspectos Conceituais em Telessaúde. Telessaúde: um instrumento de suporte assistencial e educação permanente, 2006.

SCHEFFER, M. et al. Demografia Médica No Brasil 2018. São Paulo: Departamento de Medicina Preventiva da Faculdade de Medicina da USP, 2018. 286 p. Disponível em: <https://jornal.usp.br/wp-content/ uploads/DemografiaMedica2018.pdf>. Acesso em: 1 out. 2018.

XING-HUA, S. et al. Design and Development of Tele-Diagnosis System of Medical Image Based on Mobile Terminal. 2014 7th International Conference on Intelligent Computation Technology and Automation, n. 20110172, p. 149-153, 2014. 